\title{
Presence and severity of dystonia and choreoathetosis overflow movements in participants with dyskinetic cerebral palsy and their relation with functional classification scales
}

\author{
Inti Vanmechelen 1 * Saranda Bekteshi 1 Kyra Bossier 2 Hilde Feys 2 Jan Deklerck 1 Elegast \\ Monbaliu \\ 12 \\ 1Department of Rehabilitation Sciences, KU Leuven, Bruges, Belgium; \\ 2Department of Rehabilitation Sciences, KU Leuven, Leuven, Belgium \\ Supplemental data for this article can be accessed here. \\ *CONTACT Inti Vanmechelen Inti.vanmechelen@kuleuven.be Department of Rehabilitation Sciences, KU Leuven, \\ Spoorwegstraat 12, 8200 Bruges, Belgium
}

\section{ABSTRACT}

Background: This cross-sectional study aims to investigate the presence and severity of overflow movements of dystonia and horeoathetosis in dyskinetic cerebral palsy (CP) and to assess the relationship of overflow movements with functional classification scales.

Methods: Fifty-two subjects with dyskinetic CP were included. Presence and severity of dystonia and choreoathetosis overflow movements were assessed with the Dyskinesia Impairment Scale. Functional abilities were classified with the Gross Motor Function Classification System, Manual Ability Classification System, Viking Speech Scale, Communication Function Classification System, and Eating and Drinking Ability Classification System.

Results: Dystonia and choreoathetosis overflow movements were simultaneously present. Median scores of dystonia overflow movements were significantly higher than choreoathetosis overflow movements. Dystonia and choreoathetosis overflow movements were significantly higher in extremities than in the central body. Correlations between dystonia and choreoathetosis overflow movements were fair. Moderate to good correlations were found between dystonia overflow score and Gross Motor Function Classification System, Manual Ability Classification System, and Eating and Drinking Ability Classification System.

Conclusions: This is the first study to assess overflow movements in dyskinetic CP. All participants presented with dystonia and choreoathetosis overflow movements, with higher values for dystonia overflow movements. Dystonia overflow movements seem to have a larger impact on functional abilities.

Implications for rehabilitation

- Dystonia and choreoathetosis overflow movements are both present in children with dyskinetic cerebral palsy, with dystonia overflow movements being more severe than choreoathetosis overflow movements.

- Overflow movements impact heavily on daily functional ability and the execution of voluntary activities.

- Dystonia overflow movements show good correlations with functional classification scales.

- The measurable characteristics of overflow movements can be used as a guideline for targeted treatment with, e.g., botulinum-toxin-A injections.

KEYWORDS Movement patterns; movement disorders; children; youth; functional classification scales; disability 


\section{Introduction}

Cerebral palsy (CP) describes a group of permanent disorders of the development of movement and posture, causing activity limitation, that are attributed to nonprogressive disturbances that occurred in the developing fetal or infant brain [1]. It is the most frequent cause of severe physical disability in childhood [2]. In relation to the dominant motor abnormalities, CP is subdivided into three groups: spastic, dyskinetic, and ataxic group [3]. Dyskinetic $C P$ is highly correlated with basal ganglia and thalamus lesions and is the most disabling form of $C P$ [4]. Dyskinetic CP is characterized by abnormal postures or movements associated with impaired muscle tone regulation, movement control, and coordination and comprises two major movement disorder patterns, i.e., dystonia and choreoathetosis [2].

Dystonia refers to abnormal postures and repetitive movements due to intermittent muscle contractions [5] and is commonly triggered by voluntary movements which may be aggravated by distraction, overuse, fatigue, or pain [5]. Choreoathetosis is a hyperkinetic movement disorder with fluctuating tone $[5,6]$ and can be further subdivided into chorea and athetosis. Chorea is characterized by ongoing movements with randomly appearing sequences [5,6]. Choreatic movements are rapid, involuntary, jerky, and often fragmented [6]. Athetosis is characterized by involuntary, discrete, slow, continuously changing, writhing, or contorting movements $[5,6]$. Both movement disorders are independently present in dyskinetic CP [7], but dystonia predominates in most patients [7].

Overflow muscle activation or movements are a common clinical sign in multiple neurological disorders and are also described in childhood movement disorders as dystonia [5,8]. Overflow movements are described as unintentional contractions in muscles different from the muscles normally activated for a goal-directed action [8-10]. Dystonic overflow movements commonly occur at the peak of dystonic movements [8], but they do not seem to have a purpose in the primary voluntary movement of the patient [11]. Their expression may, however, disturb functional activities and limit the execution of daily life activities.

The phenomenon has been previously assessed in focal dystonia [12,13] and Huntington's disease [14], however, much less is known about its presence and severity in dyskinetic CP [8]. Recent hypotheses in the pathophysiology of dystonia and dystonia overflow movements focus on a combination of three aspects: loss of inhibition [15], sensory dysfunction, and impaired plasticity in basal ganglia circuits $[2,15,16]$. An imbalance between the direct (control of voluntary activity) and indirect (inhibition of involuntary activity) pathways can cause a lack of inhibition, leading to excessive movements [16]. Furthermore, the increasingly used Hypertonic Assessment Tool [17] is discriminating the presence of dystonia with other hypertonic movement disorders, based on increased involuntary movements or postures with purposeful movement of a distant body part or overflow movements [18]. To date, overflow movements patterns, either for dystonia or choreoathetosis, have never been mapped before in dyskinetic CP. However, in view of therapeutic management it would be of great interest to map these clinically distinct patterns and increase the understanding in their

presence, distribution, and severity.

Therefore, the aim of this study is to gain a better understanding of overflow movements in children with dyskinetic CP by (1) investigating the presence, severity, and distribution of dystonia and choreoathetosis overflow movements and (2) assessing the relationship between the severity of dystonia and choreoathetosis overflow movements and functional classification scales in children with dyskinetic CP. The hypotheses are that (i) overflow movements will be present in both dystonia and choreoathetosis in children with dyskinetic CP and (ii) severity of overflow movements are related to severity of functional classification scales.

\section{Materials and methods Participants}

All participants were recruited from five Flemish special education schools for people with motor disabilities. Participants were screened for eligibility and diagnosed by a pediatric neurologist. Inclusion criteria were (1) aged between 5 and 22 years, (2) diagnosed with dyskinetic CP, and (3) able to understand test instructions. Exclusion criteria were orthopedic and/or neurosurgical interventions in the previous 12 months. Ethical approval was obtained from the institutional ethics committee and written informed consent was obtained from all participants or the participant's parents. 


\section{Assessment}

Overflow movements were assessed with the Dyskinesia Impairment Scale (DIS), which has proven good reliability and validity in previous measures of dystonia and choreoathetosis $[6,19]$. This video-based scale consists of two subscales, both evaluating duration and amplitude of dystonia and choreoathetosis over 12 body regions during rest and voluntary activities. This scale can also be used to evaluate dystonia and choreoathetosis overflow movement (Supplementary Figure S1). Based on expert opinion and consensus of the developers of the Dyskinesia Impairment Scale, overflow movements are scored in the 12 body regions linked to predefined voluntary activities of the Dyskinesia Impairment Scale [6]. Thirteen tasks were chosen to reflect overflow movements in adjacent or opposite limbs (Table 1). A score from 0 to 4 is given for duration and amplitude for dystonia and choreoathetosis, respectively, during two voluntary activities. Total dystonia and choreoathetosis overflow movements are the sum of the total score for duration and amplitude and are expressed as percentages. Unless otherwise specified, the overflow percentages in the results section represent the total overflow percentage, i.e., sum of duration and amplitude.

Table 1. Task with corresponding body region to be scored for overflow movements.

\begin{tabular}{|ll|}
\hline Tasks to be performed with their respective body region that was scored for overflow movement. \\
\hline Task & Scored body region \\
\hline Eating a cookie (sitting position) & Eyes \\
Opening and closing mouth repeatedly (sitting position) & Eyes \\
Moving a cup from left to right with left hand (sitting position) & Mouth \\
Answering questions (speech) (sitting position) & Neck \\
Grabbing a pen with right hand (supine lying) & Trunk \\
Grabbing a pen with right hand (supine lying) & Proximal part of the left arm \\
Grabbing a pen with right hand (supine lying) & Distal part of the left arm \\
Grabbing a pen with left hand (supine lying) & Proximal part of the right arm \\
Grabbing a pen with left hand (supine lying) & Distal part of the right arm \\
Heel-toe tap with right foot (sitting position) & Proximal part of the left leg \\
Heel-toe tap with right foot (sitting position) & Distal part of the left leg \\
Heel-toe tap with left foot (sitting position) & Proximal part of the right leg \\
Heel-toe tap with left foot (sitting position) & Distal part of the right leg \\
\hline
\end{tabular}

To grade gross motor function, manual abilities, communication and eating and drinking ability, five corresponding functional classification scales were used: the Gross Motor Function Classification System [20], Manual Ability Classification System [21], Communication Function Classification System [22], Viking Speech Scale [23], and Eating and Drinking Ability Classification System [24].

\section{Procedure}

One assessor (EM) videotaped all participants with the Dyskinesia Impairment Scale video protocol in their natural environment at the special education school. Evaluation of functional levels was done at the same day as the video recording. The scoring of dystonia and choreoathetosis overflow movement levels was performed by another assessor (KB) after training with a golden rater (EM).

\section{Statistical analysis}

Shapiro-Wilk tests showed that most variables were not normally distributed. Therefore, nonparametric statistics were used. To map the clinical patterns, the Wilcoxon signed-rank test was used to compare overflow movement scores between different regions of the body and between dystonia and choreoathetosis overflow movements. The relationship between dystonia and choreoathetosis overflow movements, different body regions and the relationship between overflow movements and functional classification scales was assessed with the Spearman's rank correlation coefficients ( $r s)$. The correlation coefficient was considered an excellent relationship above 0.75 , moderate to good between 0.50 and 0.75 , fair between 0.20 and 0.50 and values between 0.00 and 0.25 indicated no relationship [25]. Level of statistical significance was set at 0.05 and statistical analysis was performed with SPSS 24.0 (SPSS Inc., Chicago, IL). 


\section{Results}

\section{Participants}

This study included 52 children aged between 5 and 22 years ( 30 males; 22 females; mean age 14 y 6 mo; SD 4 y $1 \mathrm{mo}$ ). The distribution of the patients throughout the functional classification scales is presented in Table 2 .

Table 2. The distribution of the participants in the different functional classification scales $(\mathrm{N}=52)$.

\begin{tabular}{|cccccc|}
\hline & GMFCS & MACS & CFCS & EDACS & 8 \\
\hline I & 9 & 7 & 6 & 19 & 19 \\
\hline II & 4 & 4 & 20 & 12 & 11 \\
\hline III & 8 & 8 & 7 & 10 & 21 \\
\hline IV & 9 & 13 & 1 & 3 & NA \\
\hline V & 22 & 20 & 13 & 7 \\
\hline
\end{tabular}

GMSCS; Gross Motor Function Classification System. MACS; Manual Ability Classification System. CFCS;

Communication Function Classification System. EDACS; Eating and Drinking Ability Classification System. VSS;

Viking Speech Scale. I-V: Levels of the functional classification scales. NA = Not Applicable.

\section{Clinical patterns overflow movements \\ Dystonia versus choreoathetosis}

The total median overflow percentage was 54\%, interquartile range (IQR) 41-61.2\% with a score range between 6.8 and $85.8 \%$. For dystonia and choreoathetosis, the median scores were $67.0 \%$ (IQR 50.6-79.3\%; score range 9.1-89.9\%) and 39.2\% (IQR 28.7-55.1\%; score range 4.5-84.1\%), respectively.

Statistical comparisons (Table 3) showed significantly higher levels of dystonia overflow movements than choreoathetosis overflow movements in all body regions except for the eye region. The correlations between dystonia and choreoathetosis overflow movements were moderate to good for the body region of the right arm, distal extremities and eyes and fair for all other body regions, of which three did not reach significance at the 0.05-level (arm left, leg left, and mouth).

\section{Duration versus amplitude}

Excellent correlation ( $r s=0.80$ and $r s=0.86$ ) was found between duration and amplitude for both dystonia and choreoathetosis overflow movements, respectively. Amplitude levels of dystonia overflow movements were significantly higher than duration levels (72.7 versus 64.4\%; $p<0.001)$, whereas a similar tendency for significance was found for choreoathetosis overflow movement levels ( 42.0 versus $34.1 \% ; p=0.053$ ).

\section{Body regions}

The median percentages of dystonia and choreoathetosis overflow movements are presented in Figure 1.

Median levels of dystonia overflow movements exceeded $50.0 \%$ for all body regions, except for the trunk (37.5\%) (Supplementary Table S1). Extremities had higher median values $(82.3 \%)$ than the central body (a combination of trunk and face values) (52.2\%) (Table 4). Proximal parts of extremities (arms and legs) had higher median values (87.5\%) than distal parts (79.7\%). Median levels of choreoathetosis overflow movements reached or exceeded $50.0 \%$ for the mouth, eyes, neck, and distal parts of the arms (Supplementary Table S1). Other body regions had median levels ranging between 31.25 and $45.3 \%$, with the trunk as the only exception.

Both dystonia and choreoathetosis overflow movements showed no significant difference in the arms compared with the legs (Table 4). For the extremities, choreoathetosis levels were significantly higher $(p<0.01)$ in the distal parts compared with proximal parts, as opposed to dystonia overflow movements. The inverse results were found for the extremities compared to the central body parts, where dystonia overflow movements were higher for the extremities $(p<0.01)$, but no significant difference was present for choreoathetosis. Both dystonia and choreoathetosis overflow movements were higher for the face compared with the trunk $(p<0.01)$.

Overall, moderate to good correlations were found between central body and extremities (ranging from 0.51 to 0.63) for both dystonia and choreoathetosis overflow movements (Table 4). Moderate correlations were found between overflow movements in the arms and the legs (ranging from 0.30 to 0.38 ) and between proximal and distal body parts (ranging from 0.42 to 0.58 ). 
Figure 1. The median percentages (\%) of dystonia and choreoathetosis overflow movements in the different body regions.

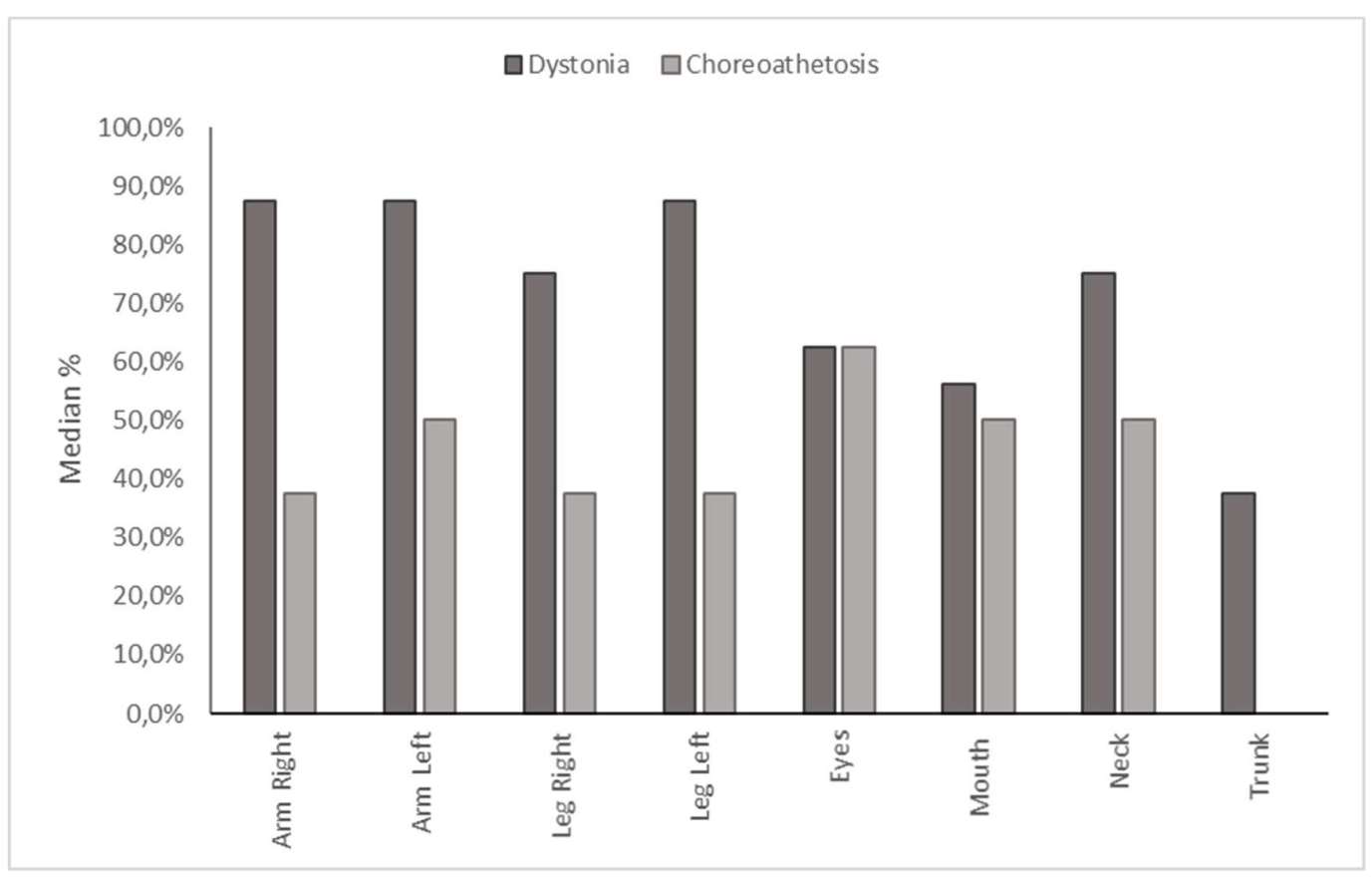

Table 3. Statistical comparison of dystonia versus choreoathetosis overflow movements in different body regions, with the median and interquartile range of the outcome measures on the Dyskinesia Impairment Scale.

\begin{tabular}{|c|c|c|c|c|c|}
\hline Overflow \% & $\begin{array}{c}\text { Median } \\
\text { (IQR) }\end{array}$ & $\begin{array}{c}\text { Median } \\
\text { (IQR) }\end{array}$ & Wilcoxon & $\begin{array}{c}\text { Spearman's rho } \\
(95 \% \text { CI) }\end{array}$ & p-value \\
\hline & Dystonia & Choreoathetosis & & & \\
\hline \multicolumn{6}{|l|}{$\underline{\text { Total levels }}$} \\
\hline Total body level & $67.0(50.6-70.3)$ & $39.2(28.7-55.1)$ & $<0.01$ & $0.44(50.13-0.67)$ & 0.001 \\
\hline Total arm level & $84.4(58.6-96.9)$ & $40.6(22.7-59.4)$ & $<0.01$ & $0.40(0.16-0.60)$ & 0.003 \\
\hline Total leg level & $75.0(50.0-93.8)$ & $37.5(14.1-62.5)$ & $<0.01$ & $0.32(0.05-0.56)$ & 0.020 \\
\hline Central body level & $52.5(38.1-65)$ & $40.0(27.5-54.4)$ & $<0.01$ & $0.29(0.02-0.55)$ & 0.039 \\
\hline \multicolumn{6}{|l|}{ Extremities } \\
\hline Arm right & $87.5(46.9-93.8)$ & $37.5(25.0-62.5)$ & $<0.01$ & $0.33(0.06-0.55)$ & 0.017 \\
\hline Arm left & $87.5(52.5-100)$ & $50.0(14.1-68.8)$ & $<0.01$ & $0.19(-0.08-0.45)$ & 0.160 \\
\hline Leg right & $75.0(50.0-100)$ & $37.5(0.0-75.0)$ & $<0.01$ & $0.40(0.10-0.60)$ & 0.004 \\
\hline Leg left & $87.5(53.1-100)$ & $37.5(0.0-62.5)$ & $<0.01$ & $0.26(-0.03-0.54)$ & 0.067 \\
\hline Proximal & $87.5(62.5-100)$ & $31.3(0.0-62.5)$ & $<0.01$ & $0.10(-0.21-0.39)$ & 0.494 \\
\hline Distal & $79.7(44.5-93.8)$ & $43.8(25.8-61.7)$ & $<0.01$ & $0.50(0.28-0.68)$ & $<0.01$ \\
\hline \multicolumn{6}{|l|}{ Central body } \\
\hline$\overline{\text { Trunk }}$ & $37.5(0.0-62.5)$ & $0.0(0.0-37.5)$ & 0.003 & $0.32(0.06-0.58)$ & 0.020 \\
\hline Face & $57.8(41.4-71.9)$ & $45.3(31-59.4)$ & 0.007 & $0.30(-0.01-0.58)$ & 0.033 \\
\hline Eyes & $62.5(0.0-75.0)$ & $62.5(25.0-75.0)$ & 0.172 & $0.50(0.23-0.73)$ & $<0.01$ \\
\hline Mouth & $56.3(37.5-75)$ & $50.0(31.25-67.2)$ & 0.043 & $0.25(-0.02-0.50)$ & 0.072 \\
\hline Neck & $75.0(50.0-87.5)$ & $50.0(0.0-62.5)$ & $<0.01$ & $0.12(-0.17-0.42)$ & 0.411 \\
\hline
\end{tabular}


Table 4. Statistical comparison of dystonia and choreoathetosis overflow movements in different body regions, with the median and interquartile range of the outcome measures on the Dyskinesia Impairment Scale.

\begin{tabular}{|c|c|c|c|c|c|}
\hline Overflow \% & $\begin{array}{l}\text { Median } \\
\text { (IQR) }\end{array}$ & $\begin{array}{c}\text { Median } \\
\text { (IQR) }\end{array}$ & Wilcoxon & $\begin{array}{c}\text { Spearman's rho } \\
(95 \% \mathrm{CI})\end{array}$ & p-value \\
\hline & Leg & Arm & & & \\
\hline Dystonia & $75.0(50.0-93.8)$ & $84.4(58.6-96.9)$ & 0.240 & $0.30(0.04-0.54)$ & 0.029 \\
\hline \multirow[t]{2}{*}{ Choreoathetosis } & $37.5(14.1-62.5)$ & $40.6(22.7-59.4)$ & 0.564 & $0.38(0.15-0.58)$ & 0.005 \\
\hline & Proximal & Distal & & & \\
\hline Dystonia & $87.5(62.5-100)$ & $79.7(44.5-93.8)$ & 0.324 & $0.42(0.13-0.67)$ & 0.002 \\
\hline \multirow[t]{2}{*}{ Choreoathetosis } & $31.3(0.0-62.5)$ & $43.8(25.8-61.7)$ & $<0.01$ & $0.58(0.35-0.75)$ & $<0.01$ \\
\hline & Central body & Extremities & & & \\
\hline Dystonia & $52.5(38.1-65.0)$ & $82.3(53.1-91.7)$ & $<0.01$ & $0.51(0.31-0.68)$ & $<0.01$ \\
\hline \multirow[t]{2}{*}{ Choreoathetosis } & $40.0(27.5-54.4)$ & $40.6(20.8-58.9)$ & 0.675 & $0.63(0.39-0.79)$ & $<0.01$ \\
\hline & Trunk & Face & & & \\
\hline Dystonia & $37.5(0.0-62.5)$ & $57.8(41.4-71.9)$ & $<0.01$ & $0.38(0.14-0.60)$ & 0.006 \\
\hline Choreoathetosis & $0.0(0.0-37.5)$ & $45.3(31.3-59.4)$ & $<0.01$ & $0.32(0.06-0.55)$ & 0.022 \\
\hline
\end{tabular}

\section{Relationship of dystonia and choreoathetosis overflow movements with functional classification scales} The relationship between functional classification levels and dystonia and choreoathetosis overflow movement scores is visualized as box plots in Figures 2 and 3.

A moderate to good correlation was found between Gross Motor Function Classification System levels and total dystonia overflow movement scores ( $r s=0.59 ; 95 \% \mathrm{Cl} 0.34-0.78 ; p<0.001$ ) and between Manual Ability Classification System severity and total dystonia overflow movements ( $r s=0.60 ; 95 \% \mathrm{Cl} 0.35-0.77 ; p<0.001$ ). For the Viking Speech Scale and the Communication Function Classification System, a fair correlation was found between both functional classification scales and total dystonia overflow movements $(\mathrm{rs}=0.43 ; 95 \% \mathrm{Cl} 0.15-$ $0.64 ; p=0.001$ and $r s=0.34 ; 95 \% \mathrm{Cl} 0.08-0.58 ; p=0.015)$. A good to moderate correlation was found between the Eating and Drinking Ability Classification System and total dystonia overflow movements $(\mathrm{rs}=0.52 ; 95 \% \mathrm{Cl}$ $0.25-0.73 ; p<0.001)$.

The total choreoathetosis overflow movement scores showed fair correlation with the Gross Motor Function Classification System levels ( $r \mathrm{~s}=0.38 ; 95 \% \mathrm{Cl} 0.07-0.63 ; \mathrm{p}=0.005$ ) and Manual Ability Classification System levels ( $r s=0.41 ; 95 \% \mathrm{Cl} 0.15-0.65 ; p=0.003$ ). No correlation was found between total choreoathetosis overflow movement scores and Viking Speech Scale ( $r s=0.27 ; 95 \% \mathrm{Cl}-0.01$ to $0.51 ; p=0.054$ ) and Communication Function Classification System ( $r s=0.08 ; 95 \% \mathrm{Cl}-0.19$ to $0.35 ; \mathrm{p}=0.561$ ). Fair correlations were found for the Eating and Drinking Ability Classification System ( $r s=0.38 ; 95 \% \mathrm{Cl} 0.08-0.63 ; \mathrm{p}=0.006)$. 
Figure 2. Median and interquartile range of the dystonia overflow movement levels according to the Gross Motor Function Classification System (GMFCS), the Manual Ability Classification System (MACS), the Viking Speech Scale (VSS), the Communication Function Classification System (CFCS), and Eating and Drinking Ability Classification System (EDACS).
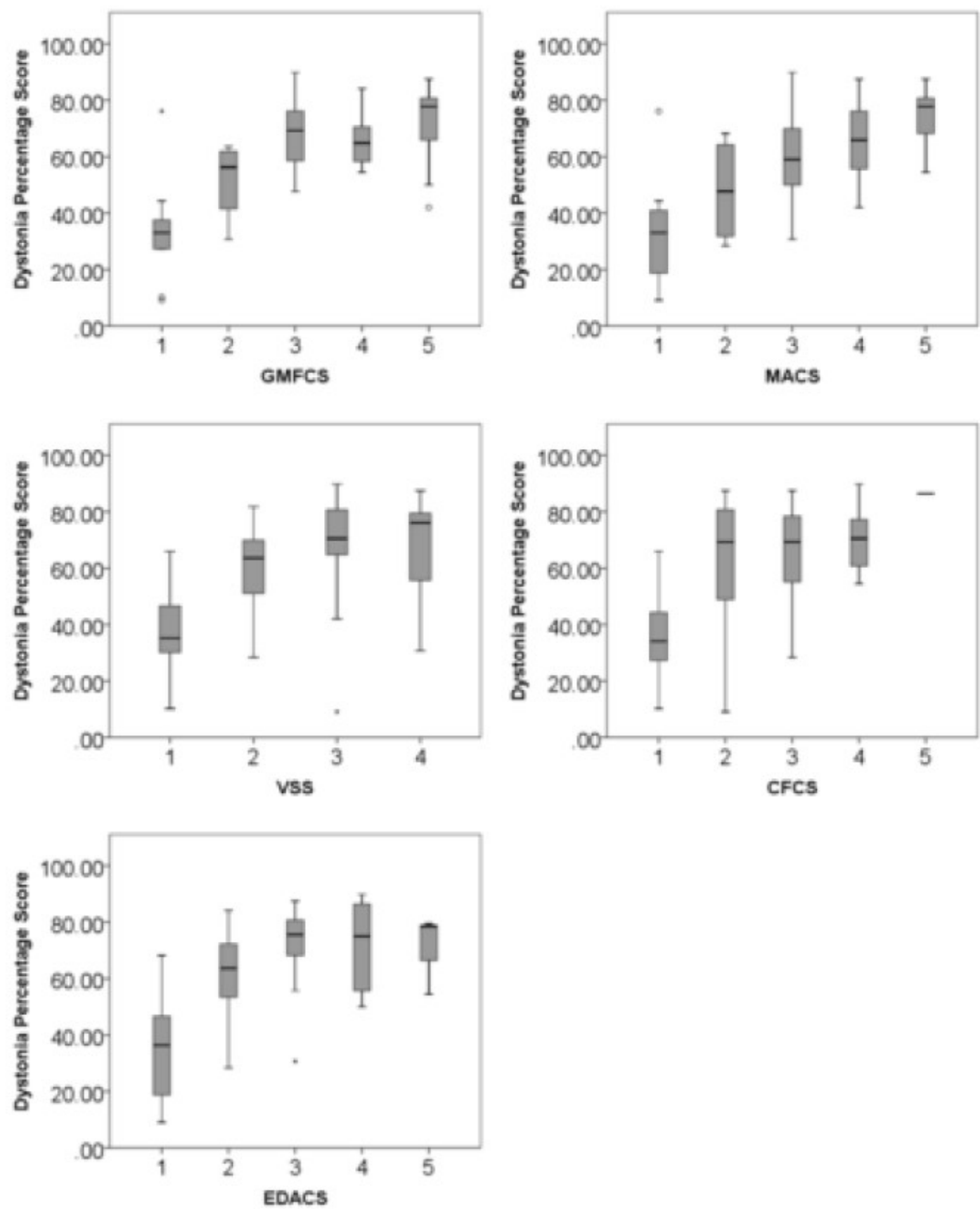
Figure 3. Median and interquartile range of the choreoathetosis overflow movement levels according to the Gross Motor Function Classification System (GMFCS), the Manual Ability Classification System (MACS), the Viking Speech Scale (VSS), the Communication Function Classification System (CFCS), and Eating and Drinking Ability Classification System (EDACS).
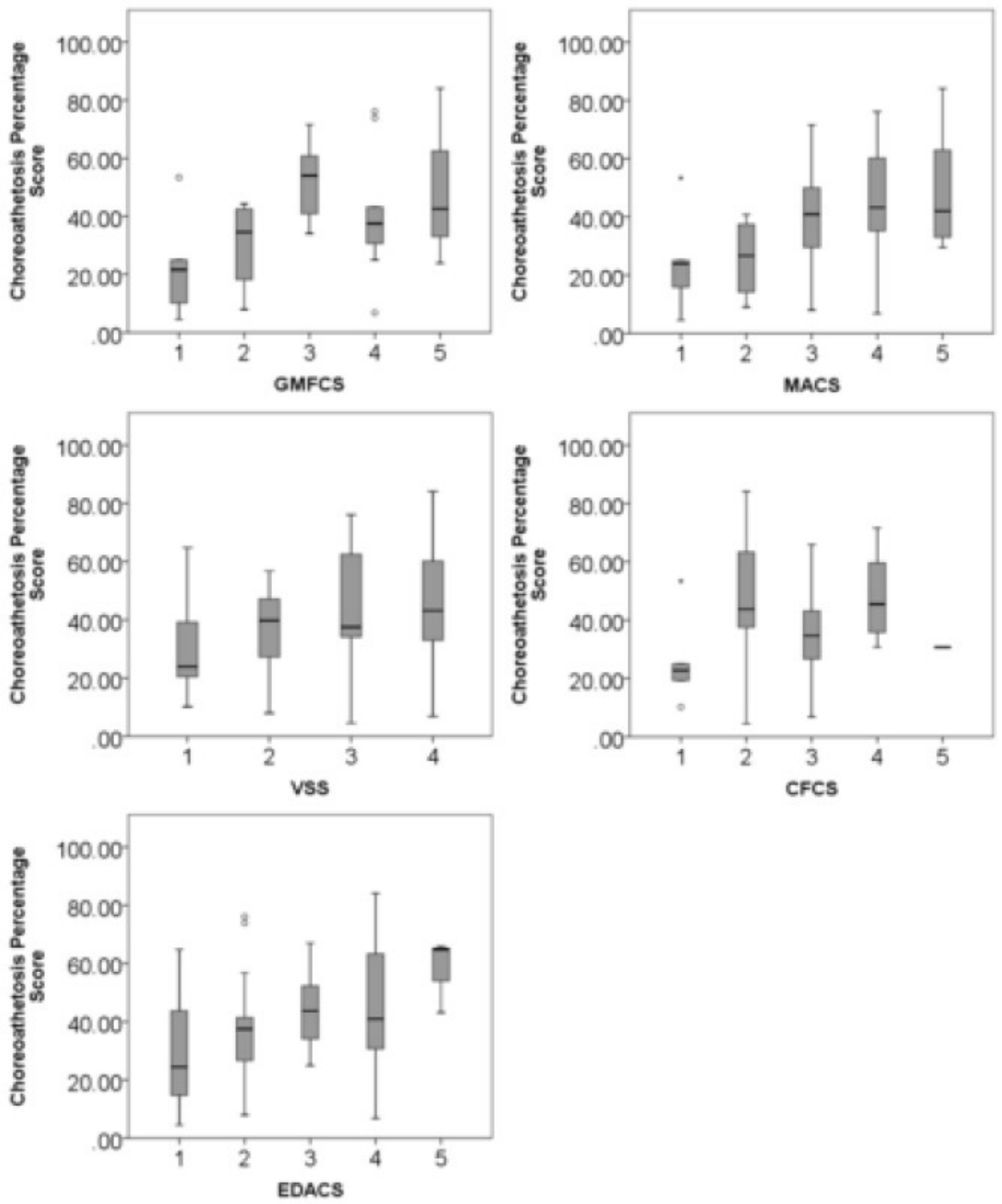


\section{Discussion}

The overall aim of this retrospective study was to gain insight in the clinical patterns of overflow movements in children with dyskinetic CP and its relationship with functional ability. Overflow movements are commonly associated with dystonia, but their prevalence and clinical expression remain unknown. To the best of our knowledge, this is the first study to map overflow movements for both dystonia and choreoathetosis in children with dyskinetic $C P$.

The first principal aim of this study was to investigate the presence of dystonia and choreoathetosis overflow movements in dyskinetic CP and to map the clinical patterns over several body regions. A simultaneous occurrence of dystonia and choreoathetosis overflow movements was found in all participants. Severity of overflow movements for dystonia was significantly higher compared to choreoathetosis, with median percentage levels of 67.0 and $39.2 \%$, respectively. Similar patterns for dystonia and choreoathetosis were previously reported [7].

Interestingly, for dystonia overflow movement levels, significant higher levels of amplitude were found compared with duration and a similar tendency toward significant higher levels of amplitude was found for choreoathetosis overflow movements. These findings are in disagreement with previous findings in dyskinetic $\mathrm{CP}$ [7] wherein duration levels were significantly higher than amplitude levels. Our results might be different from previous research because overflow movements are only measured during activity and do not include rest postures. Inherently to the lack of coordination in dystonia and choreoathetosis, extreme positions (e.g., full flexion or extension) are clinically more seen and may be a causing factor of this finding. The higher levels of amplitude in overflow movements emphasize their disruptive nature and their negative impact on the execution of voluntary activities. For future interventions, higher levels of amplitude overflow movements could provide a rationale to attempt limb-specific interventions (e.g., orthotics) or cognitive training [26] to reduce the amplitude of overflow movements and subsequently increase the success rate for the execution of activities of daily living.

In the extremities, dystonia overflow movements were significantly higher than choreoathetosis overflow movements for all limbs. These results implicate that dystonia overflow movements are more severe than choreoathetosis overflow movements, which emphasizes the more disabling aspect of dystonia and dystonia overflow movements. Correlations between dystonia and choreoathetosis overflow movements ranged from poor to fair, underscoring the independent occurrence of both dystonia and choreoathetosis overflow movements and the importance of their differentiation for further management. Also in the central body, trunk and mouth, dystonia overflow movements were significantly higher than choreoathetosis overflow movements, with trunk choreoathetosis overflow movements being zero. The overall low values for trunk overflow movements may be explained by the fact that the trunk is a more rigid body part and therefore less prone to exhibit involuntary movements. For the eye region, dystonia and choreoathetosis overflow movement scores were equal. These values may be explained by the difficulty of scoring movement disorders in the eye region, as this region is inherently characterized by movements, thereby challenging the differentiation of dystonia from choreoathetosis [27].

When comparing the different body regions, no significant difference between arms and legs overflow movements for either dystonia or choreoathetosis was found, whereas a higher percentage of both dystonia and choreoathetosis was previously found in the arms compared to the legs [7]. This discrepancy could be explained by the fact that overflow movements are not perceived as voluntary movements and their presence may therefore be less prominent in the arm region. Choreoathetosis overflow movements were higher in the distal extremities than proximal, whereas no significant difference was present for dystonia overflow movements. Dystonia overflow movements were found to be higher in the extremities than in the central body, whereas no significant difference was present for choreoathetosis overflow movements. This is an unexpected finding since we expected choreoathetosis overflow movements to be equally higher in the extremities. However, choreoathetotic movements are not triggered by voluntary attempts to the same degree as dystonic movements [5] and the resting scores of the Dyskinesia Impairment Scale are not taken into account in overflow scoring. This could possibly explain for the lack of difference in choreoathetosis overflow movements. 
The second aim of this study was to assess the relationship between the severity of dystonia and choreoathetosis overflow movements and functional classification scales during voluntary activities. Moderate to good correlations were found between total dystonia overflow movements level and Gross Motor Function Classification, Manual Ability Classification System, Eating and Drinking Ability Classification System, and fair correlations between total dystonia overflow movements level and Viking Speech Scale and Communication Function Classification System. This implies that dystonia overflow movements were more numerously present in more severely affected children, a phenomenon that is also seen in Huntington's disease [14]. Reported correlations were overall slightly lower than previously published in the dyskinetic CP population [28] implying that dystonia overflow movements seem to be less disabling than the presence of dystonia itself. However, significant associations were found with all classification scales, emphasizing the importance of dystonia overflow movements in daily living activities. In particular, a good correlation was found between dystonia overflow movement percentage and the Manual Ability Classification System and the Gross Motor Function Classification System, underlining the disabling aspects of overflow movements. Previous research in focal dystonia showed that overflow in focal hand dystonia can be used to differentiate between dystonic and secondary compensatory movements, which serves to increase accuracy during therapeutic botulinum toxin injections into dystonic forearm muscles.

Correlations between total choreoathetosis overflow movement scores and functional classification scales were fair for the Gross Motor Function Classification System, Manual Ability Classification System, and Eating and Drinking Ability Classification System. The correlations between choreoathetosis overflow movement percentage and functional classification scales are overall higher than previously reported for choreoathethosis percentages [28]. Specifically for the Gross Motor Function Classification System and the Manual Ability Classification System, the fair correlations suggest that choreoathetosis overflow movements may have a more severe impact than the choreoathetosis percentages as previously reported. These results suggest that the presence of choreoathetosis and choreoathetosis overflow movements may be distinct clinical patterns and may need to be differentiated for further specification of treatment management plans. Further research is necessary to investigate the predictive characteristic of dystonia and choreoathetosis overflow movements in the rehabilitation and management of dyskinetic $\mathrm{CP}$.

The predominance of dystonia overflow movements and its moderate to good correlation with functional classification scales may suggest that treatment of dystonia overflow movements could lead to a greater effect on functional abilities compared to treatment of choreoathetosis overflow movements. The high levels of dystonia and choreoathetosis overflow movements found in this study is a clinical phenomenon that should be taken into account during therapy, especially in the arm region as significantly higher levels of dystonia and choreoathetosis overflow movements were found in this body region. The effect of therapy on overflow movements in dyskinetic $\mathrm{CP}$ has yet to be researched.

This study has limitations. First, the age range of 5 to 22 years is large, and no distinction between age groups was made. For straightforward conclusions and generalization of these results to the complete dyskinetic CP population, a greater sample size is necessary. Second, notwithstanding the considerably large study sample size for this population, there were often few participants in some levels of the Gross Motor Function Classification System, Manual Ability Classification System, Viking Speech Scale, Communication Function Classification System, and Eating and Drinking Ability Classification System. This was especially the case in the Communication Function Classification System, where the participants were not equally distributed throughout the different levels and further statistical analysis was therefore not possible.

Despite these shortcomings, this study was the first to map the clinical patterns of dystonia and choreoathetosis overflow movements in a large cohort of participants with dyskinetic CP and to relate the overflow movement levels to functional classification scales. 


\section{Conclusions}

This is the first study to assess overflow movements in dyskinetic CP. Dystonia and choreoathetosis overflow movements were found to be simultaneously present with a clear predominance of dystonia overflow movements in the majority of our participants. The impact of dystonia overflow movements on functional abilities is higher compared with choreoathetosis overflow movements. The findings of this study contribute to a better understanding of dystonia and choreoathetosis overflow movements and their mutual relationship. Increased insights in dystonia and choreoathetosis and their clinical expression are indispensable for the evaluation and enhancement of rehabilitation and management strategies in people with dyskinetic CP.

\section{Acknowledgments}

We would like to thank all the participants and their parents for their time and effort to participate in this study. Special thanks to the Committee of Flemish Motor Disability Centers (KOMPAS).

\section{Disclosure statement}

No potential conflict of interest was reported by the authors.

\section{References}

1. Rosenbaum P, Paneth N, Leviton A, et al. A report: the definition and classification of cerebral palsy April 2006. Dev Med Child Neurol Suppl. 2007;109:8-14.

2. Monbaliu E, Himmelmann K, Lin J-P, et al. Clinical presentation and management of dyskinetic cerebral palsy. Lancet Neurol. 2017;16:741-749.

3. Cans Christine, Dolk H, Platt MJ, et al. Recommendations from the SCPE collaborative group for defining and classifying cerebral palsy. Dev Med Child Neurol. 2007;49:35-38.

4. Krageloh-Mann I, Cans C. Cerebral palsy update. Brain Dev. 2009;31:537-544.

5. Sanger TD, Chen D, Fehlings DL, et al. Definition and classification of hyperkinetic movements in childhood. Mov Disord. 2010;25:1538-1549.

6. Monbaliu Elegast, Ortibus ELS, de Cat JOS, et al. The Dyskinesia Impairment Scale: a new instrument to measure dystonia and choreoathetosis in dyskinetic cerebral palsy. Dev Med Child Neurol. 2012;54:278-283.

7. Monbaliu E, de Cock $P$, Ortibus $E$, et al. Clinical patterns of dystonia and choreoathetosis in participants with dyskinetic cerebral palsy. Dev Med Child Neurol. 2016;58:138-144.

8. Albanese A, Bhatia K, Bressman SB, et al. Phenomenology and classification of dystonia: a consensus update. Mov Disord. 2013;28:863-873.

9. Addamo PK, Farrow M, Hoy KE, et al. The effects of age and attention on motor overflow production - a review. Brain Res Rev. 2007;54:189-204.

10. Hoy KE, Fitzgerald PB, Bradshaw JL, et al. Investigating the cortical origins of motor overflow. Brain Res Rev. 2004;46:315-327.

11. Licari MK, Billington J, Reid SL, et al. Cortical functioning in children with developmental coordination disorder: a motor overflow study. Exp Brain Res. 2015;233:1703-1710.

12. Cox BC, Cincotta M, Espay AJ. Mirror movements in movement disorders: a review. Tremor Other Hyperkinet Mov. 2012;2.

13. Young SJ, van Doornik J, Sanger TD. Finger muscle control in children with dystonia. Mov Disord. 2011;26:1290-1296.

14. Georgiou-Karistianis N. Motor overflow in Huntington's disease. J Neurol Neurosurg Psychiatry. 2004;75:904-906.

15. Hallett M. Neurophysiology of dystonia: the role of inhibition. Neurobiol Dis. 2011;42:177-184.

16. Quartarone A, Hallett M. Emerging concepts in the physiological basis of dystonia. Mov Disord.

2013;28:958-967.

17. Knights S, Datoo N, Kawamura A, et al. Further evaluation of the scoring, reliability, and validity of the Hypertonia Assessment Tool (HAT). J Child Neurol. 2014;29:500-504.

18. Jethwa Anita, Mink Jonathan, Macarthur Colin, et al. Development of the Hypertonia Assessment Tool (HAT): a discriminative tool for hypertonia in children. Dev Med Child Neurol. 2009;52:e83-e87.

19. Monbaliu E, Ortibus E, Prinzie P, et al. Can the Dyskinesia Impairment Scale be used by inexperienced raters? A reliability study. Eur J Paediatr Neurol. 2013;17:238-247.

20. Palisano R, Rosenbaum P, Walter S, et al. Development and reliability of a system to classify gross motor function in children with cerebral palsy. Dev Med Child Neurol. 1997;39:214-223. 
21. Eliasson A-C, Krumlinde-Sundholm L, Rösblad B, et al. The Manual Ability Classification System (MACS) for children with cerebral palsy: scale development and evidence of validity and reliability. Dev Med Child Neurol. 2006;48:549.

22. Hidecker Maryjocooley, Paneth Nigel, Rosenbaum Peterl, et al. Developing and validating the Communication Function Classification System for individuals with cerebral palsy. Dev. Med Child Neurol. 2011;53:704-710.

23. Pennington L, Virella D, MjØen T, et al. Development of The Viking Speech Scale to classify the speech of children with cerebral palsy. Res Dev Disabil. 2013;34:3202-3210.

24. Sellers D, Mandy A, Pennington L, et al. Development and reliability of a system to classify the eating and drinking ability of people with cerebral palsy. Dev Med Child Neurol. 2014;56:245-251.

25. Portney LG, Watkins MP. Foundations of clinical research: applications to practice. Upper Saddle River (NJ): Pearson/Prentice Hall; 2009.

26. Gimeno H, Polatajko HJ, Cornelius V, et al. Protocol for N-of-1 trials with replications across therapists for childhood-onset dystonia rehabilitation: Study 2 [Protocole des essais a effectif unique avec repetitions par differents ergotherapeutes pour la readaptation de la dystonie debutant dans l'enfance: Etude 2]. Can J Occup Ther. 2018;85:255-260.

27. Comella CL, Leurgans S, Wuu J, et al. Rating scales for dystonia: a multicenter assessment. Mov Disord. 2003;18:303-312.

28. Monbaliu E, De La Peña M-G, Ortibus E, et al. Functional outcomes in children and young people with dyskinetic cerebral palsy. Dev Med Child Neurol. 2017;59:634-640. 
Supplementary Figure S1: Structure of the Dyskinesia Impairment Scale used for dystonia and choreoathetosis overflow movements

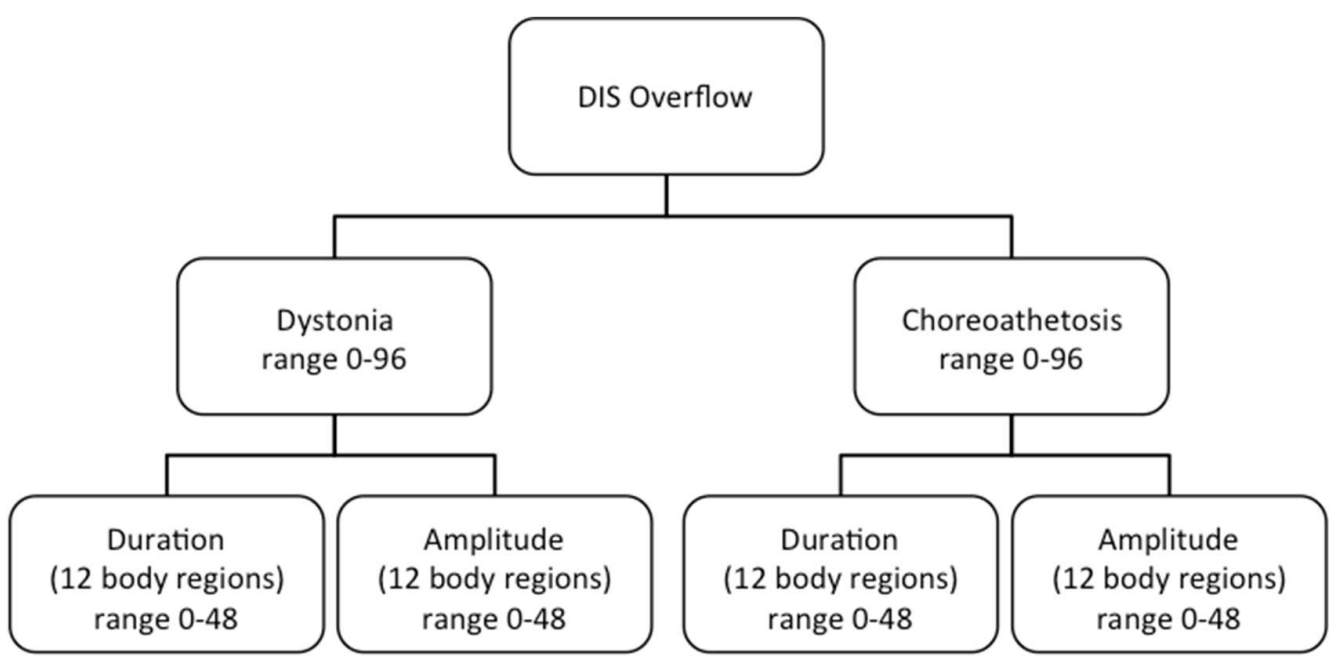

Supplementary Table S1: Descriptive statistics of dystonia and choreoathetosis

Median percentages of the Dyskinesia Impairment Scale overflow scores for the body regions

\begin{tabular}{|llllllll|}
\hline \multicolumn{6}{|l|}{ Median percentages of the Dyskinesia Impairment Scale overflow scores for the body regions } \\
\hline Measure & $\begin{array}{l}\text { Total } \\
\text { body }\end{array}$ & Eyes & Mouth & Neck & Trunk & Arm right & Arm left \\
\hline Dystonia & $67 \%$ & $65.5 \%$ & $56.3 \%$ & $75 \%$ & $37.5 \%$ & $87.5 \%$ & $87.5 \%$ \\
\hline Choreoathetosis & $39.2 \%$ & $62.5 \%$ & $50 \%$ & $50 \%$ & $0 \%$ & $37.5 \%$ & $50 \%$ \\
\hline & Leg right & Leg left & $\begin{array}{l}\text { Arm right } \\
\text { proximal }\end{array}$ & $\begin{array}{l}\text { Arm right } \\
\text { distal }\end{array}$ & $\begin{array}{l}\text { Arm left } \\
\text { proximal }\end{array}$ & $\begin{array}{l}\text { Arm left } \\
\text { distal }\end{array}$ & \\
\hline Dystonia & $75 \%$ & $87.5 \%$ & $87.5 \%$ & $87.5 \%$ & $87.5 \%$ & $100 \%$ & \\
\hline Choreoathetosis & $37.5 \%$ & $37.5 \%$ & $31.3 \%$ & $56.3 \%$ & $43.8 \%$ & $56.3 \%$ \\
\hline
\end{tabular}

